WHO-Ziel nicht erreicht

\title{
Beim Masernschutz hinkt Deutschland hinterher
}

- Die Elimination der Masern ist ein erklärtes Ziel der WHO. Gefordert wird eine Inzidenz von höchstens einer Erkrankung pro 1000000 Einwohner pro Jahr.

Doch von diesem Ziel ist Deutschland weit entfernt, und zwar in allen Altersgruppen bis zum 39. Lebensjahr. Insgesamt wurden im Jahr 2010 in Deutschland 777 Masernfälle ge-

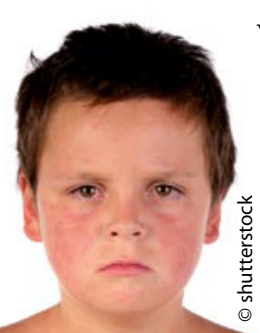

von 95\% der Bevölkerung notwendig.

Die weiterhin hohe Inzidenz veranlasste die STIKO im letzten Jahr dazu, die Impfempfehlung zu erweitern. Die Masernimpfung wird nun für alle nach 1970 geborenen Erwachsenen empfohlen, sofern sie nicht oder nur einmal geimpft sind oder der Impfstatus unklar ist. Um den Impfschutz bei Jugendlichen zu vervollständigen, sollen alle im Kleinkindalter versäumten Impfungen vor dem meldet. Um die Masern auszurotten, dürften hierzulande höchstens 85 Fälle pro Jahr auftreten. Hierzu wäre eine Durchimpfungsrate
18. Geburtstag nachgeholt werden. JN .

- Epidemisches Bulletin 32/2010; Epidemisches Bulletin 3/2011

Krankheitsausbrüche

\section{Große Impflücken bei Asylbewerbern}

- Vergangenes Jahr hat es in Deutschland mehrfach Ausbrüche von Krankheiten, gegen die geimpft werden kann, in Asylbewerber-Unterkünften gegeben. Es gab sowohl einen Varizellenausbruch in einem Heim in München als auch Masernausbrüche in Unterkünften in Neumünster und Karlsruhe. Schließlich ist auch Hepatitis A vermehrt bei Flüchtlingen aus Afghanistan aufgetreten.

Das verdeutlicht: Bei erst kürzlich in Deutschland angekommenen Menschen ist der Impfschutz lückenhaft. Dr. Günter Pfaff vom Landesgesundheitsamt Baden-Württemberg erinnert an die ethische Pflicht, bei Migranten zeitnah nach ihrer Ankunft auf einen guten Impfstatus hinzuwirken.

EIS -

- 2. Nationale Impfkonferenz, Stuttgart, 8.-9.

Februar 2011

\section{Neuer Info-Flyer}

\section{Was tun, wenn unvollständige Impfungen lange zurückliegen?}

Leserfrage: Ein Patient (Jahrgang 1966) hat folgende dokumentierte Impfungen: Gegen Tetanus wurde er am 5.8. und 5.9.1969 sowie am 10.4.1972 geimpft, außerdem am 6.10. und 4.11.1988. Aktuell ist er noch einmal am 15.2.2011 wegen einer Verletzung geimpft worden. Gegen Polio wurde er dreimal geimpft (20.11.1969, 19.1.1970, 13.11.1975).

Er hat bisher keine Impfung gegen Diphtherie und Pertussis bekommen. Keine Grundimmunisierung wurde abgeschlossen. Laut STIKO zählt ja jede Impfung. Welches Vorgehen empfehlen Sie? Soll ich jetzt IPV impfen und in zehn Jahren $\mathrm{Td}+\mathrm{aP}$ oder in vier Wochen Td, IPV + aP, oder eher für den Diphtherieschutz Grundimmunisierung mit $\mathrm{Td}$, einmalig mit aP und IPV?

Antwort von Dr. Christine Nagler, Offenbach: Leider gibt es keinen mo- novalenten Impfstoff gegen Pertussis mehr, sodass Sie im vorliegenden Fall einen Kombinationsimpfstoff verwenden sollten. Um die fehlende Grundimmunisierung gegen Diphtherie zu beginnen, würde ich sofort einmal gegen Diphtherie impfen. Vier Wochen später erfolgt eine Impfung gegen Tetanus, Diphtherie, Keuchhusten und Polio.

Bitte weisen Sie den Patienten darauf hin, dass der Kombinationsimpfstoff nicht zur Grundimmunisierung zugelassen ist und dass es durch die erneute Tetanusimpfung zu einer verstärkten lokalen Reaktion kommen könnte.

Alternativ können Sie auch einen Impfstoff gegen Tetanus, Diphtherie und Pertussis verwenden und gegen Polio separat impfen. In sechs bis zwölf Monaten zum Abschluss der DiphtherieGrundimmunisierung sollte dann noch eine Diphtherie-Impfung erfolgen. \section{zu HPV}

- Vertreter des Berufsverbandes der Frauenärzte e. V. und andere Experten beklagen die niedrige HPV-Impfrate in Deutschland. Hierzulande ist nur jedes dritte Mädchen gegen HPV geimpft, in Ländern wie England, Schottland oder Spanien sind es dagegen rund 90\%. Die HPV-Impfung wird seit mehr als zwei Jahren von der STIKO für Mädchen zwischen 12 und 17 Jahren empfohlen.

Die Aufklärung über Gebärmutterhalskrebs wird jetzt durch einen neuen Info-Flyer unterstützt. Er kann kostenlos angefordert werden: Zervita-Geschäftsstelle, Universitätsklinikum Tübingen, Elfriede-Aulhorn-Str. 6, 72076 Tübingen, Tel. $07071 / 2980196 \quad$ JN .

- Informationen des Deutschen Grünen Kreuzes, AG Zervixkarzinom 